\title{
Evidências de Validade do Índice de Religiosidade de Duke (P-DUREL) entre Adolescentes
}

\author{
Miriam Raquel Wachholz Strelhow ${ }^{1}$, Jorge Castellá Sarriera \\ Universidade Federal do Rio Grande do Sul, Porto Alegre-RS, Brasil
}

\section{RESUMO}

O objetivo deste estudo foi verificar as propriedades psicométricas da versão brasileira do Índice de Religiosidade de Duke (P-DUREL) entre adolescentes. Por meio de uma plataforma on-line, participaram 1.248 adolescentes brasileiros de ambos os sexos, entre $12 \mathrm{e}$ 18 anos $(M=15,09, D P=1,77)$, sendo 57,3\% meninas. A estrutura fatorial foi verificada através de análises fatoriais exploratória e confirmatória. O modelo unidimensional apresentou índices de ajuste satisfatórios, bem como adequada confiabilidade $(\alpha=0,874)$. Análises multigrupo por sexo e idade indicaram que a estrutura fatorial e os pesos fatoriais são semelhantes para os grupos testados. A escala apresentou alta correlação positiva com o Módulo "Espiritualidade, religiosidade e crenças pessoais" (WHOQOL-SRPB). Conclui-se que o instrumento é adequado para a avaliação da religiosidade entre adolescentes brasileiros, podendo ser utilizado em futuros estudos com essa população.

Palavras-chave: religiosidade; validade estatística; psicometria; DUREL.

\section{ABSTRACT - Validity evidence of the Duke Religion Index (P-DUREL) among adolescents}

The purpose of this study was to verify psychometric properties of the Brazilian version of the Duke Religion Index (P-DUREL) among adolescents. Through an online platform, 1,248 Brazilian adolescents of both genders, between 12 and 18 years of age $(M=15.09$, $S D=1.77$ ) participated, $57.3 \%$ of whom were female. The factorial structure was verified through exploratory and confirmatory factor analyses. The one-dimensional model presented satisfactory fit indexes, as well as adequate reliability $(\alpha=0.874)$. Multigroup analyses by sex and age indicated that the factorial structure and factorial weights are similar for the groups tested. The scale presented a high positive correlation with the Module "Spirituality, religiousness and personal beliefs" (WHOQOL-SRPB). It is concluded that the instrument is suitable for the evaluation of religiousness among Brazilian adolescents, and may be used in future studies with this population.

Keywords: religiousness; statistical validity; psychometry; DUREL.

\section{RESUMEN - Evidencias de validez del Índice de Religiosidade de Duke (P-DUREL) entre adolescentes}

El objetivo de este estudio fue verificar las propiedades psicométricas de la versión brasileña del Índice de Religiosidad de Duke (P-DUREL) entre adolescentes. A través de una plataforma online, participaron 1.248 adolescentes brasileños de ambos sexos, entre 12 y 18 años $(M=15.09, D S=1.77)$, un 57,3\% del sexo femenino. La estructura factorial fue verificada a través de análisis factoriales exploratorios y confirmatorios. El modelo unidimensional presentó índices de ajuste satisfactorios, así como confiabilidad adecuada $(\alpha=0,874)$. Los análisis multigrupo por sexo y edad indicaron que la estructura factorial y los pesos factoriales son similares para los grupos testados. La escala presentó una alta correlación positiva con el Módulo "Espiritualidad, religiosidad y creencias personales" (WHOQOL-SRPB). Como conclusión, se puede afirmar que el instrumento es adecuado para la evaluación de la religiosidad entre adolescentes brasileños, pudiendo ser utilizado en futuros estudios con esta población.

Palabras clave: religiosidad; validez estadística; psicometría; DUREL.

Tem crescido o interesse em pesquisas sobre religiosidade e espiritualidade, especialmente sua relação com o bem-estar e questões de saúde (Lucchetti, Lucchetti, \& Vallada, 2013; Strelhow \& Henz, 2017). De acordo com Koenig, McCullough e Larson (2001), enquanto a religião se refere ao sistema organizado de crenças, práticas, rituais e símbolos, que facilitam a relação com o sagrado (Deus, força maior, verdade suprema), a religiosidade indica o quanto uma pessoa acredita, segue e pratica uma determinada religião. Já a espiritualidade se refere a uma busca pessoal pela compreensão de questões existenciais maiores como, por exemplo, o fim e o sentido da vida, e suas relações com o sagrado e/ou transcendente.

Muitos instrumentos foram desenvolvidos para a avaliação da religiosidade e outros aspectos relacionados e estão disponíveis em diferentes países, principalmente na língua inglesa (Lucchetti et al., 2013; Marques \& Aguiar, 2014). Entretanto, o mesmo não pode ser afirmado para

${ }^{1}$ Endereço para correspondência: Rua Ramiro Barcelos, 2600, Santa Cecília, 90035-003, Porto Alegre, RS. Tel.: (11) 96638-7972. E-mail: raquelwch@gmail.com Artigo derivado da tese de doutorado da primeira autora, com a orientação do segundo autor. Pesquisa financiada pelo Conselho Nacional de Desenvolvimento Científico e Tecnológico - CNPq 
instrumentos disponíveis no Brasil, especialmente considerando seu uso com adolescentes. Observa-se a necessidade de instrumentos que permitam avançar nos estudos com essa população.

Lucchetti et al. (2013) realizaram um levantamento sistemático dos instrumentos disponíveis em português para avaliar espiritualidade e religiosidade. Os resultados apontaram que a maioria dos instrumentos foi validada ou criada a partir de 2005 , sendo que o primeiro artigo publicado foi em 2002, em Portugal. Esses dados demonstram que as pesquisas sobre esses construtos ainda estão muito recentes nos países de língua portuguesa. Os autores encontraram 20 instrumentos: $45 \%$ avaliavam religiosidade, $40 \%$ espiritualidade, $10 \%$ coping (enfrentamento) religioso/espiritual e 5\% espiritualidade e religiosidade. Do total, 15 foram traduzidos e três criados para o português no Brasil, enquanto dois foram para o português de Portugal. Mas os autores destacam que alguns desses instrumentos não possuem relato de propriedades psicométricas nas amostras brasileiras, e a maioria dos instrumentos apresenta índices de confiabilidade satisfatórios, mas não apresentam dados de validade concorrente e divergente e/ou teste-reteste.

Ressalta-se, entretanto, que a grande parte desses instrumentos foi criada ou adaptada para populações adultas. São raros os instrumentos e os estudos que se dedicam à avaliação desses aspectos na infância e adolescência. Pode-se citar como exemplo de exceção, o instrumento criado para jovens e adolescentes (14 a 24 anos) na Pesquisa sobre a Juventude Brasileira (Cerqueira-Santos \& Koller, 2009). Nessa pesquisa, foram incluídas sete questões de religiosidade, referentes à importância da religião, prática religiosa, e busca a Deus e a instituição religiosa em momentos de dificuldade. Essas questões foram computadas em um índice único formando uma Escala de Religiosidade com sete itens. No estudo de Jahn e Dell '́́glio (2017) a escala apresentou consistência interna de 0,86 (alfa de Cronbach).

Destaca-se também a Escala de Coping Religioso para Crianças (CRC), traduzida e adaptada para o Brasil (Strelhow, Bedin, \& Sarriera, 2017). O instrumento, composto por 28 itens representando as dimensões de coping religioso positivo e negativo, apresentou boas propriedades psicométricas na amostra brasileira. A dimensão Coping Religioso Positivo $(\alpha=0,91)$ pode ser avaliada em três fatores: crença no suporte de Deus, busca pela instituição religiosa e intercessão. $\mathrm{E}$ a dimensão de Coping Religioso Negativo $(\alpha=0,80)$, que também pode ser avaliada em três fatores: descontentamento com Deus ou outros; reavaliação negativa do significado e reavaliação de punição.

Sobre as duas escalas citadas acima, observa-se que a primeira reúne diferentes aspectos da prática religiosa em uma medida unidimensional, enquanto a segunda avalia especificamente o uso do coping religioso. Outros estudos realizados com adolescentes brasileiros optaram por medidas de afiliação religiosa (ter ou não uma religião) para a avaliação de aspectos da religiosidade, como o estudo de Silva, Giordani e Dell'Àglio (2017). Dessa forma, entende-se que é necessária a disponibilização de mais instrumentos que possam abranger a religiosidade entre adolescentes em suas diferentes dimensões. Um instrumento que tem sido bastante utilizado a âmbito internacional e também tem se mostrado promissor em estudos no Brasil, é o Índice de Religiosidade da Universidade de Duke (DUREL; Koenig \& Büssing, 2010).

Original dos Estados Unidos, esse instrumento avalia a religiosidade a partir da diferenciação em três dimensões: religiosidade organizacional, que se refere à prática pública, social e institucional, como participar de encontros religiosos (por exemplo, cultos, missas, grupos de oração); religiosidade não organizacional para referir-se a atividade religiosa privada, pessoal e individual (como orar/rezar, estudar as escrituras, assistir programas de TV religiosos), e a religiosidade intrínseca, relacionada ao grau de compromisso ou motivação religiosa pessoal (Koenig \& Büssing, 2010). As primeiras duas dimensões são avaliadas por meio de itens únicos, enquanto para a religiosidade intrínseca foram formulados três itens.

A escala foi traduzida para o português brasileiro, sendo aqui denominada de P-DUREL (MoreiraAlmeida, Peres, Aloe, Lotufo Neto, \& Koenig, 2008). Estudos de validação já foram feitos com diferentes amostras de adultos brasileiros: uma amostra de baixa renda da cidade de São Paulo (Lucchetti et al., 2012); uma amostra de estudantes universitários da área da Saúde e outra de pacientes psiquiátricos (Taunay et al., 2012); amostra de usuários de serviços públicos de saúde, em Ribeirão Preto, São Paulo (Martinez et al., 2014); e por meio de uma plataforma virtual (de Paula, 2015). A partir desses estudos, o instrumento demonstrou boa consistência interna, confiabilidade teste-reteste (Coeficiente de Correlação Intraclasse $>0,90$ ), bem como adequada validade convergente-discriminante entre adultos. Em estudos internacionais com adolescentes a escala também tem demonstrado boa confiabilidade (Barkin, Miller, \& Luthar, 2015; Nabipour, Khanjani, Nakhaee, Moradlou, \& Sullman, 2015).

Os autores recomendam o uso das subescalas separadas do instrumento, visto que cada dimensão pode apresentar implicações distintas e, por vezes, até opostas a aspectos da saúde (Koenig \& Büssing, 2010). Essa estrutura foi testada e tem sido utilizada em muitas pesquisas (Lace \& Handal, 2018; Tabrizi et al., 2017; Wang, Rong, \& Koenig, 2014). Entretanto, outros estudos têm verificado um funcionamento diferente da escala. Por exemplo, Cruz et al. (2017) encontraram uma estrutura bifatorial, composta por um fator de religiosidade intrínseca, e outro de práticas religiosas (que inclui tanto a prática organizacional como a não organizacional). Também estudos recentes têm apontado para a unidimensionalidade do instrumento, com a existência de uma variável 
latente "religiosidade" que englobaria as três dimensões (Chen, Wang, Phillips, Sun, \& Cheng, 2014; de Paula, 2015; Saffari, Zeidi, Pakpour, \& Koenig, 2013; Thomas, McDaniel, Albright, Fletcher, \& Koenig, 2018).

O presente estudo tem como objetivo verificar as propriedades psicométricas da escala P-DUREL em uma amostra de adolescentes brasileiros. Para tanto, se propõe a investigar sua estrutura fatorial, sua consistência interna, e sua validade convergente com o Módulo "Espiritualidade, religiosidade e crenças pessoais" (WHOQOL-SRPB, Fleck, Borges, Bolognesi, \& Rocha, 2003).

\section{Método}

\section{Participantes}

O presente estudo foi realizado por meio de uma plataforma on-line desenvolvida especialmente para esse fim. A amostra foi composta por voluntários que aceitaram participar da pesquisa através de convites on-line. Dessa forma, a amostra foi selecionada por conveniência. Os participantes foram 1.248 adolescentes brasileiros de ambos os sexos, com idades entre 12 e 18 anos $(M=15,09, D P=1,77)$, sendo $57,3 \%$ meninas.

Do total de 26 Estados brasileiros, participaram adolescentes de 22 estados e mais do Distrito Federal, sendo que os que tiveram maior número de participantes foram: Rio Grande do Sul $(n=828,66,3 \%)$, São Paulo $(n=171,13,7 \%)$, Rio de Janeiro $(n=96,7,7 \%)$, Paraná $(n=27,2,2 \%)$ e Distrito Federal $(n=24,1,9 \%)$. As características relacionadas às crenças religiosas ou espirituais são apresentadas na Tabela 1. Destaca-se que 51,8\% dos adolescentes afirmaram que têm uma religião e a praticam, sendo que $37,8 \%$ se declararam católicos e $28,9 \%$ evangélicos tradicionais.

Tabela 1

Características da Amostra em Relação a Crenças Espirituais e Religiosas

\begin{tabular}{lcc}
\multicolumn{1}{c}{ Variável } & Frequência \\
\cline { 2 - 3 } Crenças religiosas e espirituais & $n$ \\
Acredito em forças superiores (não necessariamente em Deus) & 175 & 14,0 \\
Tenho uma religião, mas não a prático & 314 & 25,2 \\
Tenho uma religião e a prático & 647 & 51,8 \\
Ateu & 54 & 4,3 \\
Agnóstico & 58 & 4,6 \\
Religião Declarada & & 37,8 \\
Católica & 436 & 11,4 \\
Evangélico - Pentecostal & 132 & 28,9 \\
Evangélico - Tradicional & 334 & 8,0 \\
Espírita & 92 & 2,7 \\
Umbandismo & 31 & 5,3 \\
Não tenho crenças religiosas e espirituais & 61 & 3,9 \\
Tenho várias afiliações religiosas & 45 & 2,0 \\
Outras & 23 & \\
\hline
\end{tabular}

\section{Instrumentos}

Os adolescentes responderam a um questionário estruturado composto por dados sociodemográficos (idade, ano escolar, afiliação religiosa), bem como o P-DUREL e o Módulo Espiritualidade, Religiosidade e Crenças Pessoais (WHOQOL-SRPB) da Organização Mundial de Saúde. As informações das escalas serão explicitadas a seguir:

P-DUREL (Índice de Religiosidade de Duke).

$\mathrm{O}$ instrumento foi originalmente desenvolvido nos Estados Unidos (Koenig, Parkerson, \& Meador, 1997), composto por cinco itens que, segundo os autores, representam três dimensões da religiosidade: um item para comportamento religioso organizacional, um item para comportamento religioso não organizacional, e três itens para religiosidade intrínseca. O P-DUREL já passou pelo processo de tradução e adaptação para o Brasil (MoreiraAlmeida et al., 2008), e sua versão em português tem apresentado boas propriedades psicométricas. No estudo de Taunay et al. (2012) o instrumento foi avaliado em uma amostra composta de estudantes universitários da área da Saúde $(n=323)$ com alfa total de 0,89 , e para a subescala religiosidade intrínseca um $\alpha=0,87$. E em uma amostra de pacientes psiquiátricos $(n=102)$ com alfa total de 0,87 e para a subescala religiosidade intrínseca $\alpha=0,81$. Também foram encontrados bons índices de teste-reteste e validade convergente e divergente com instrumentos de espiritualidade e depressão (Taunay et al. 2012). 
Os itens das dimensões de religiosidade organizacional e não organizacional são avaliados em uma escala de frequência tipo Likert de 1 (Mais do que uma vez por semana; mais do que uma vez ao dia) a 6 (Nunca), enquanto os itens de religiosidade intrínseca são respondidos em uma escala Likert de cinco pontos, variando de 1 (Totalmente verdade para mim) a 5 (Não é verdade).

Módulo "Espiritualidade, religiosidade e crenças pessoais" (WHOQOL-SRPB). Integrante do questionário de qualidade de vida da Organização Mundial de Saúde. O instrumento foi desenvolvido a partir de um estudo multicêntrico conduzido em 18 centros em 15 países, incluindo o Brasil (Fleck et al., 2003). O WHOQOLSRPB é composto por 32 itens, divididos em oito dimensões (quatro itens em cada uma): conexão a ser ou força espiritual; sentido na vida; admiração; totalidade e integração; força espiritual; paz interior; esperança e otimismo; e fé. Os itens são respondidos em uma escala tipo Likert de cinco pontos. No estudo de validação, o WHOQOLSRPB em português apresentou confiabilidade através do $\alpha$ de Cronbach entre 0,72 e 0,95 para os domínios do WHOQOL-SRPB, e 0,96 para o índice geral (Panzini, Maganha, Rocha, Bandeira, \& Fleck, 2011). Com a amostra do presente estudo, o instrumento apresentou confiabilidade adequada, sendo $\alpha=0,96$ para a escala geral, e as dimensões variando de 0,68 a 0,94 .

\section{Estudo piloto}

Inicialmente foi realizado um procedimento para validação semântica do instrumento. Este foi respondido individualmente por 20 adolescentes selecionados por conveniência, com o objetivo de verificar a compreensão em relação aos itens da escala e possíveis dificuldades em relação a eles. Os adolescentes não apresentaram dúvidas em relação a P-Durel. Após essa etapa, o instrumento foi colocado em plataforma on-line, por meio de um site criado especificamente para a presente pesquisa.

$\mathrm{O}$ instrumento on-line foi então submetido a uma aplicação piloto, do qual participaram 81 adolescentes de 12 a 18 anos, sendo 54,3\% meninas. A coleta de dados foi realizada em duas escolas da Região Metropolitana de Porto Alegre, uma particular e uma pública, após a concordância institucional, com o preenchimento do Termo de Concordância Institucional, a autorização dos pais (TCLE) e o assentimento dos alunos, confirmado no termo desenvolvido no próprio site da pesquisa. $\mathrm{O}$ instrumento foi respondido pelos alunos no laboratório de informática, em turno escolar.

Com o estudo piloto foi possível verificar o funcionamento do site, bem como verificar a fidedignidade dos instrumentos. A escala P-Durel composta por cinco itens obteve uma boa consistência interna $(\alpha=0,87)$.

\section{Procedimentos de coleta de dados}

A coleta de dados foi realizada de forma online por meio de um formulário eletrônico. Os participantes foram convidados a participar da pesquisa através de redes sociais virtuais e de e-mails. Ainda, para atingir um maior número de participantes foi realizada a divulgação da pesquisa por meio de convites e cartazes em escolas e instituições religiosas. As pessoas que se interessaram pela pesquisa escolheram responder ao questionário de forma espontânea.

\section{Procedimentos de análise dos dados}

Inicialmente foi feita a verificação da frequência de missing. O valor máximo de missing nos itens foi de $3 \%$. Dessa forma, optou-se por fazer a imputação por regressão dos dados faltantes. Para a sequência das análises, a amostra foi dividida ao meio. Com a primeira metade $(n=625)$ foi realizada análise fatorial exploratória para verificar a estrutura fatorial da escala, por meio do Programa Factor. Conforme sugestão de Damásio (2012), optou-se pelo procedimento de análise paralela, sendo a matriz de dispersão calculada a partir de correlações de Pearson, e tendo o Unweighted Least Squares como método de estimação indicado. Para a análise da consistência interna da escala, foram considerados os valores do $\alpha$ de Cronbach, calculado com o auxílio do programa SPSS 21.

Para verificar o modelo unifatorial do instrumento, optou-se pela análise fatorial confirmatória e análises multigrupo por sexo e idade, com a segunda metade da amostra $(n=623)$, com o auxílio do software AMOS 21. $\mathrm{Na}$ avaliação dos modelos, foram considerados o qui-quadrado, o Índice de Comparação do Ajuste (CFI), além do TLI (Índice de Tucker e Lewis), NFI (Índice de Ajuste Normatizado), e a análise dos resíduos com o Erro Quadrático Médio de Aproximação (RMSEA) com seus intervalos de confiança (Byrne, 2010). Para esses índices a literatura indica como valores aceitáveis para o CFI, TLI e NFI acima de 0,90, sendo melhores os valores próximos a 1, e para o RMSEA indicam-se como valores ideais abaixo de 0,08 (Hair, Black, Babin, Anderson, \& Tatham, 2009). Para as análises multigrupo, considerou-se como índice comparativo entre os modelos, a diferença entre os CFIs dos modelos testados $(\Delta \mathrm{CFI})$ que não pode ser maior que 0,01 para indicar comparabilidade entre os modelos (Milfont \& Fisher, 2010). Para a comparação por idade, a amostra foi dividida em três grupos: 12-13, 14-15 e 16-18 anos.

Para a avaliação da validade convergente, utilizou-se os coeficientes de correlação de Pearson, entre a escala, suas dimensões (propostas pelos autores) e um instrumento que avalia espiritualidade, religiosidade e crenças pessoais (WHOQOL-SRPB). Para interpretação das correlações, utilizou-se os seguintes valores: correlações baixas entre 0,20 e 0,40, correlações moderadas entre 0,40 e 0,60 e altas entre 0,60 e 0,80 (Bisquerra, Sarriera, \& Martínez, 2004). 


\section{Procedimentos Éticos}

O projeto desta pesquisa foi aprovado pelo Comitê de Ética em Pesquisa do Instituto de Psicologia da Universidade a qual os autores estão vinculados. Ao acessar a página on-line da pesquisa, os participantes eram inicialmente encaminhados para o Termo de Consentimento Livre e Esclarecido (TCLE), elaborado especificamente para esta pesquisa. Neste, foi solicitado o assentimento para a participação, bem como a autorização de um responsável, no caso dos menores de 18 anos, por meio de uma identificação de endereço eletrônico (e-mail) e botão de concordância dos responsáveis. Os procedimentos estão de acordo com os critérios de ética na pesquisa com seres humanos, conforme Resolução ${ }^{\circ}$ 466/2012 do Conselho Nacional de Saúde.

\section{Resultados}

\section{Análise fatorial exploratória $(n=625)$}

De acordo com a proposta dos autores, a Escala P-DUREL engloba três aspectos importantes da religiosidade: Religiosidade Organizacional
(RO), Religiosidade Não Organizacional (RNO) e Religiosidade Intrínseca. Os dois primeiros aspectos são avaliados a partir de itens únicos, enquanto a terceira dimensão é composta por um fator, com três itens. Optou-se por realizar uma análise fatorial exploratória, com o objetivo de verificar a formação fatorial do instrumento a partir dos dados empíricos com adolescentes brasileiros.

Inicialmente foram analisados os pressupostos para as análises, onde se verificou que foram atendidos, com uma boa medida de adequação da amostra (Kaiser-Meyer-Olkin $=0,862$ ) e o teste de esfericidade de Bartlett altamente significativo $(p<0,001)$. A análise fatorial exploratória foi realizada utilizando-se o procedimento de análise paralela, sendo a matriz de dispersão calculada a partir de correlações de Pearson, e tendo o Unweighted Least Squares como método de estimação indicado. A análise apontou para uma formação unifatorial, reunindo os cinco itens em um único fator. Esse valor obteve um autovalor de 3,41 e uma variância explicada de $77,10 \%$. A Tabela 2 indica a carga fatorial de cada um dos itens.

Tabela 2

Análise Fatorial Exploratória da P-DUREL $(n=625)$

\begin{tabular}{lc}
\hline & Carga Fatorial \\
\hline Religiosidade Organizacional (RO) & 0,716 \\
Com que frequência você vai a uma igreja, templo ou encontro religioso? & \\
Religiosidade Não Organizacional (RNO) & 0,710 \\
$\quad$ Com que frequência você dedica o seu tempo a atividades religiosas individuais, & \\
como preces, rezas, meditações, leitura da bíblia ou de outros textos religiosos? & 0,785 \\
Religiosidade Intrínseca (RI) & 0,815 \\
1. Em minha vida, eu sinto a presença de Deus (ou do Espírito Santo) & 0,850 \\
2. As minhas crenças religiosas estão realmente por trás de toda a minha maneira de viver & 77,10 \\
3. Eu me esforço para viver a minha religião em todos os aspectos da vida &
\end{tabular}

\section{Análise da consistência interna}

A consistência interna da P-Durel foi verificada por meio do alfa de Cronbach. A análise revelou que a confiabilidade se mostrou adequada para a amostra $(\alpha=0,874)$. Verificou-se individualmente o alfa para a escala, bem como caso cada item fosse deletado, mas todos os itens demonstraram contribuir ao instrumento.

\section{Análise fatorial confirmatória $(n=623)$}

Visto que a análise fatorial Exploratória indicou uma estrutura unifatorial, e estudos anteriores encontraram um modelo unidimensional satisfatório para a escala, optou-se por verificar por meio de análise fatorial confirmatória esse modelo unidimensional, no qual se entende que há um fator latente "religiosidade" que explica os cinco itens da escala. Não foi possível testar o modelo trifatorial indicado por Koenig e Büssing (2010), porque os dois primeiros fatores são compostos por apenas um item, o que resulta em um modelo não estimável. A Figura 1 mostra o modelo unidimensional, com os parâmetros padronizados.

O primeiro modelo testado alcançou índices de ajustes satisfatórios (CFI $>0,90)$. Entretanto, o resíduo RMSEA ficou alto, acima de 0,08. Optou-se por acrescentar uma covariância entre os erros 1 e 2, dos itens de religiosidade organizacional e não organizacional, indicados por meio dos índices de modificação. A partir dessa modificação, vê-se que os índices de ajuste melhoraram, assim como o índice de resíduo (Tabela 3). 


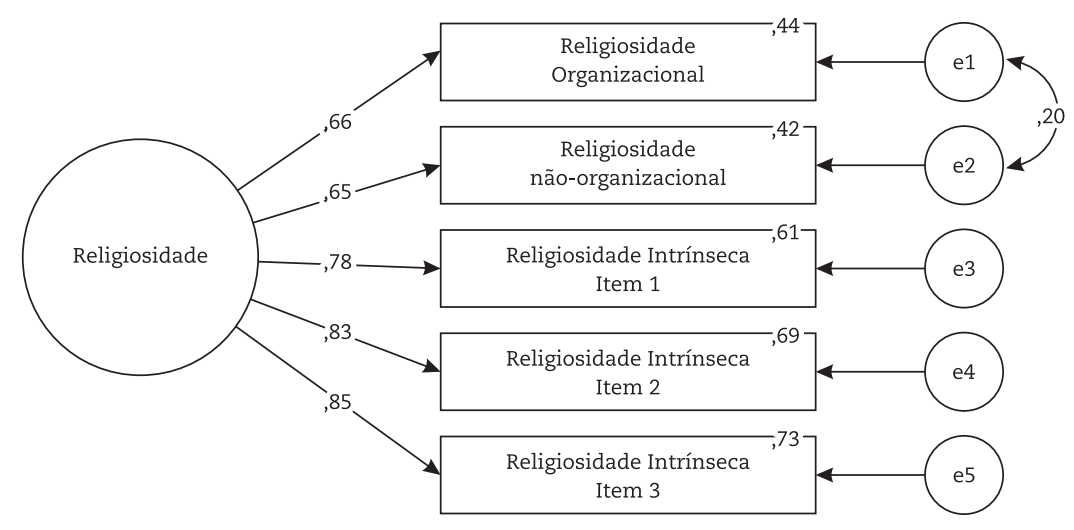

Figura 1. Modelo unidimensional da escala P-Durel, com cargas padronizadas

Tabela 3

Índices de Ajuste da Análise Fatorial Confirmatória da P-DUREL

\begin{tabular}{lccccccc}
\hline & $\chi^{2}$ & $d f$ & $p$ & NFI & TLI & CFI & RMSEA (IC $)^{\text {a }}$ \\
\hline 1. P-DUREL Modelo Unifatorial & 34,992 & 5 & $<0,001$ & 0,977 & 0,960 & 0,980 & $0,098(0,069-0,130)$ \\
2. P-DUREL com covariância entre erros & 15,043 & 4 & 0,005 & 0,990 & 0,982 & 0,993 & $0,067(0,033-0,104)$ \\
\hline
\end{tabular}

Nota. Valores de referência: CFI, NFI e TLI>0,90; RMSEA<0,08; IC $=$ =Intervalo de Confiança de 95\%

\section{Análise multigrupo por sexo e idade}

Para verificar se a estrutura fatorial da escala é a mesma para meninos e meninas e para as diferentes idades, optou-se pela análise multigrupo, a partir de dois modelos: no primeiro todos os parâmetros estão livremente estimados para os grupos. No segundo, as cargas fatoriais são restringidas para serem iguais entre os grupos, testando sua invariância.

Em relação ao sexo, para o modelo livremente estimado a análise indicou $\chi^{2}(8)=22,861 \quad(p=0,004)$, $\mathrm{CFI}=0,990 \quad$ e $\quad \mathrm{RMSEA}=0,055 \quad(\mathrm{IC}=0,029-0,082)$ e, para o modelo com as cargas fatoriais restringidas um $\chi^{2}(12)=34,746 \quad(p=0,001), \quad$ CFI $=0,985$ e RMSEA =0,055 (IC=0,034-0,077). Em relação à idade, para o modelo livremente estimado os índices de ajuste ficaram em $\chi^{2}(28)=42,516(p=0,039)$, CFI $=0,990$ e RMSEA $=0,029$ ( $\mathrm{IC}=0,007-0,046)$. Enquanto que para o modelo com as cargas fatoriais restringidas os índices foram: $\chi^{2}(32)=45,891(p=0,053), \mathrm{CFI}=0,991$ e RMSEA $=0,026(\mathrm{IC}=0,000-0,043)$. Assim, as diferenças entre os CFIs $(\Delta \mathrm{CFI}<0,01)$ por sexo e por idade indicam que tanto a estrutura fatorial é plausível para os grupos testados, como os itens possuem os mesmos significados para os grupos.

\section{Validade convergente-discriminante}

Foram avaliadas as correlações entre a escala P-Durel e a escala WHOQOL-SRPB, que avalia espiritualidade, religiosidade e crenças pessoais. Optou-se ainda por apresentar a correlação entre os escores das três dimensões da P-Durel propostas pelos autores. Como indica a Tabela 4, o cálculo feito por meio dos coeficientes de correlação de Pearson revelou correlações moderadas a altas entre os escores das dimensões do P-DUREL, indicando que há validade convergente entre elas, entretanto sem haver redundância (validade discriminante).

Tabela 4

Correlações entre as Dimensões da P-Durel e WHOQOL-SRPB

\begin{tabular}{|c|c|c|c|c|c|}
\hline$n=625$ & P-DUREL & RO & RNO & $\mathrm{RI}$ & WHOQOL-SRPB \\
\hline P-DUREL & 1 & & & & \\
\hline $\begin{array}{l}\text { Com que frequência você vai a uma igreja, } \\
\text { templo ou encontro religioso? - RO }\end{array}$ & $0,806^{* *}$ & 1 & & & \\
\hline $\begin{array}{l}\text { Com que frequência você dedica o seu temp } \\
\text { a atividades religiosas individuais, como } \\
\text { preces, rezas, meditações, leitura da bíblia } \\
\text { ou de outros textos religiosos? - RNO }\end{array}$ & $0,811^{* *}$ & $0,574^{* *}$ & 1 & & \\
\hline
\end{tabular}


Tabela 4 (continuação)

Correlações entre as Dimensões da P-Durel e WHOQOL-SRPB

\begin{tabular}{|c|c|c|c|c|c|}
\hline$n=625$ & P-DUREL & $\mathrm{RO}$ & RNO & RI & WHOQOL-SRPB \\
\hline Religiosidade Intrínseca - RI & $0,938^{* *}$ & $0,634^{* *}$ & $0,630^{* *}$ & 1 & \\
\hline WHOQOL-SRPB & $0,681^{* *}$ & $0,482^{* *}$ & $0,540^{* *}$ & $0,676^{* *}$ & 1 \\
\hline
\end{tabular}

Como se pode ver na Tabela 4, a escala P-Durel apresentou correlação positiva e significativa com a WHOQOL-SRPB $(r=0,681)$, assim como suas dimensões, considerando o modelo proposto pelos autores. A dimensão que apresentou a maior correlação foi a de Religiosidade Intrínseca $(r=0,676)$. Em relação às correlações entre as dimensões, observa-se que foram significativas e positivas entre $\mathrm{RO}$ e RI $(r=0,634), \mathrm{RO}$ e RNO $(r=0,574)$ e RI e RNO $(r=0,630)$.

\section{Discussão}

O P-DUREL é um instrumento amplamente utilizado no âmbito internacional, e também tem apresentado resultados satisfatórios em estudos com amostras brasileiras diversificadas de adultos (de Paula, 2015; Lucchetti et al., 2012; Taunay et al., 2012). As análises realizadas no presente estudo indicam o bom funcionamento do instrumento também entre adolescentes brasileiros.

$\mathrm{Na}$ análise fatorial exploratória, a solução encontrada indicou a presença de um único fator. A possibilidade da unidimensionalidade foi confirmada na análise fatorial confirmatória, e pelas altas correlações entre as dimensões. Estudos recentes também confirmaram bons índices de ajuste para o modelo unidimensional com outras amostras (Chen et al., 2014; de Paula, 2015; Saffari et al., 2013). Em contrapartida, Koenig e Büssing (2010) ressaltam que o instrumento deve ser utilizado com suas três dimensões separadamente, justamente para captar interações diferentes que cada dimensão pode ter com outros aspectos de interesse, como saúde e bem-estar. Os resultados do presente estudo indicam a possibilidade para uso do instrumento como uma dimensão global de religiosidade. Entende-se que a decisão para o uso do instrumento deve levar em conta os objetivos da pesquisa e as análises que serão realizadas (cuidando, por exemplo, com a multicolinearidade presente entre as dimensões, no caso de estudos que optarem pelo modelo com as dimensões separadas).

Por meio do alfa de Cronbach, pode-se concluir que o instrumento tem boa confiabilidade, considerando a escala como um todo. Os índices encontrados com os adolescentes brasileiros foram próximos ao estudo de Barkin et al. (2015), bem como com as amostras do estudo brasileiro de Taunay et al., (2012), e mais alta que os das amostras brasileiras de Lucchetti et al. (2012) e de Paula (2015), em que os alfas ficaram entre 0,76 e 0,80 .
Em relação à validade convergente e discriminante, optou-se no tocante estudo por avaliar as correlações entre a P-Durel, suas próprias dimensões e o escore total do WHOQOL-SRPB, procedimentos que foram adotados em estudo anterior (Taunay et al., 2012). As análises entre as dimensões da P-Durel, indicam correlação moderada entre RO e RNO, e correlações altas entre RI e RO e RI e RNO. As duas correlações altas têm valores bastante próximos, indicando que quanto maior a religiosidade intrínseca relatada pelo adolescente, maior também a frequência de sua prática religiosa, tanto organizacional como privada (bem como o inverso também). Os valores das correlações entre as dimensões estão próximos aos valores encontrados por Taunay et al. (2012), e acima dos encontrados por Martinez et al. (2014). No estudo de Taunay et al. (2012), houve maior correlação entre RI e RO, sendo menores as correlações entre RI e RNO nas duas amostras avaliadas.

Em relação à validação com o WHOQOL-SRPB, a P-Durel apresentou ccorrelação alta, positiva e significativa, indicando que quanto maior a religiosidade, maior também a espiritualidade. Em relação aos valores das correlações com as três dimensões da P-DUREL, observou-se que foram bastante próximos aos encontrados no estudo de Taunay et al. (2012). A correlação foi moderada entre o WHOQOL-SRPB e RO e RNO e alta com RI. O resultado está de acordo com o esperado, visto que RI está relacionada ao grau de motivação religiosa pessoal, indicando o quanto a crença religiosa move a pessoa, bem como o quanto ela se sente em ligação com Deus (Koenig \& Büssing, 2010) e o WHOQL-SRPB tem entre seus domínios fatores de ligação com ser ou força espiritual, bem como sobre o papel das crenças e fé na vida da pessoa (Fleck et al. 2003).

\section{Considerações Finais}

Considera-se que o P-DUREL é um instrumento que apresenta evidências de validade para ser utilizado como uma medida de religiosidade entre adolescentes brasileiros. $\mathrm{O}$ fato de ser um instrumento com poucos itens facilita seu uso em pesquisas com diferentes construtos e aplicações em grandes populações. Espera-se que esse estudo possa contribuir para a ampliação da avaliação da religiosidade entre adolescentes, e especialmente sobre a relação desta com aspectos de saúde e bem-estar, ainda pouco conhecidos na população brasileira. 
Uma limitação do instrumento, já apontada pelos próprios autores (Koenig \& Büssing, 2010), refere-se a ser designado para medir religiosidade em religiões ocidentais (especialmente a tradição cristã, mas também Judaica e Islâmica), e que pode ser menos precisa para avaliar tradições religiosas orientais, como Hinduísmo ou Budismo. Por exemplo, o instrumento refere-se a Deus (ou Espírito Santo). Entretanto, estudos realizados em diferentes culturas têm demonstrado que seu uso é válido para pessoas de diferentes religiões, fazendo-se adaptações, quando necessário (Chen et al., 2014; Nabipour et al., 2015; Saffari et al., 2013). Estudos futuros podem verificar se seu funcionamento se mantém para participantes de religiões distintas no Brasil.
Ressalta-se que embora a amostra desse estudo seja grande e composta por adolescentes de diferentes estados brasileiros, ela não é uma amostra aleatória. Todos os adolescentes que participaram tiveram acesso à internet e, por isso, não representam todos os adolescentes do país. Novos estudos podem ser feitos com pesquisas em papel, com outros grupos populacionais, bem como para ampliar a participação de adolescentes do Norte e Nordeste do Brasil. Da mesma forma, este estudo não avaliou outras evidências de validade como a estabilidade do instrumento, com teste-reteste, o que pode ser ampliado em futuros estudos com o instrumento.

\section{Referências}

Barkin, S. H., Miller, L., \& Luthar, S. S. (2015). Filling the void: Spiritual development among adolescents of the affluent. Religion and Health, 54(3), 844-861. doi: 10.1007/s10943-015-0048-z

Bisquerra, R., Sarriera, J. C., \& Martínez, F. (2004). Introdução à estatística: Enfoque informático com o pacote estatístico SPSS. Porto Alegre: Artmed. Byrne, B. M. (2010). Structural equation modeling with AMOS. Basic concepts, applications and programming (2nd ed.). New York: Routledge.

Chen, H., Wang, Z., Phillips, M. R., Sun, Y., \& Cheng, H. G. (2014). Internal consistency and test-retest reliability of the Chinese version of the 5-item Duke University Religion Index. Shanghai Arch Psychiatry, 26(5), 300-309. doi: 10.11919/j.issn.1002-0829.214088

Cerqueira-Santos, E., \& Koller, S. (2009). A dimensão psicossocial da religiosidade entre jovens brasileiros. Em R. M. C. Libório \& S. Koller (Eds), Adolescência e juventude: Risco e proteção na realidade brasileira (pp. 133-154). São Paulo: Casa do Psicólogo.

Cruz, J. P., Reyes, R. W. P., Colet, P. C., Estacio, J. C., Caldeira, S., Vitorino, L. M., \& Koenig, H. G. (2017). Psychometric evaluation of the Filipino versions of the Duke University Religion Index and the Spiritual Coping Strategies Scale in Filipino hemodialysis patients. Journal of Religion and Health, 56(4), 1381-1396. doi: 10.1007/s10943-016-0355-z

Damásio, B. F. (2012). Uso da análise fatorial exploratória em psicologia. Avaliação Psicológica, 11(2), 213-228. Recuperado de http://pepsic. bvsalud.org/scielo.php?script $=$ sci_abstract\&pid=S1677-04712012000200007

de Paula, J. J. (2015). Propriedades psicométricas do Índice de Religiosidade de Duke aplicado em plataforma virtual. Cadernos Saúde Coletiva, 23(3), 276-279. doi: 10.1590/1414-462X201500030090

Fleck, M. P. A., Borges, Z. N., Bolognesi, G., \& Rocha, N. S. (2003). Desenvolvimento do WHOQOL, módulo espiritualidade, religiosidade e crenças pessoais. Revista de Saúde Pública, 37(4), 446-455. doi: 10.1590/S0034-89102003000400009

Hair, J. F., Black, W. C., Babin, B. J., Anderson, R. E., \& Tatham, R. L. (2009). Análise multivariada de dados (6a edição). Porto Alegre: Bookman.

Jahn, G., \& Dell'Aglio, D. (2017). A religiosidade em adolescentes brasileiros. Revista de Psicologia da IMED, 9(1), 38-54. doi: 10.18256/21755027.2017.v9i1.1541

Koenig H. G., \& Büssing A. (2010). The Duke University Religion Index (DUREL): A five-item measure for use in epidemiological studies. Religions, 1(1), 78-85. doi: 10.3390/rel1010078

Koenig, H. G., McCullough, M., \& Larson, D. B. (2001). Handbook of religion and health: A century of research reviewed. New York: Oxford University Press.

Koenig, H., Parkerson, G. R., \& Meador, K. G. (1997). Religion index for psychiatric research. American Journal of Psychiatry, $154(6), 885-886$. doi: 10.1176/ajp.154.6.885b

Lace, J. W., \& Handal, P. J. (2018). Confirming the tripartite structure of the Duke University Religion Index: A methodological approach. Journal of Religion and Health, 57(2), 704-716. doi: 10.1007/s10943-017-0556-0

Lucchetti, G., Granero Lucchetti, A. L., Peres, M. F., Leão, F. C., Moreira-Almeida, A., \& Koenig, H. G. (2012). Validation of the duke religion index: DUREL (Portuguese version). Journal of Religion and Health, 51(2), 579-86. doi: 10.1007/s10943-010-9429-5

Lucchetti, G., Lucchetti, A. L., G., \& Vallada, H. (2013). Measuring spirituality and religiosity in clinical research: A systematic review of instruments available in the Portuguese language. Sao Paulo Medical Journal, 131(2), 112-22. doi: 10.1590/S1516-31802013000100022

Marques, L. F., \& Aguiar, A. P. A. (2014). Instrumentos de mensuração da religiosidade/espiritualidade (R/E) e seus construtos. Revista Pistis E Praxis, Teologia Pastoral, 6(1), 727-745. doi: 10.7213/revistapistispraxis.7678

Martinez, E. Z., Alves, A. C., Carneiro, A. F. T. M., Jorge, T. M., Carvalho, A. C. D., \& Zucoloto, M. L. (2014). Investigação das propriedades psicométricas do Duke Religious Index no âmbito da pesquisa em Saúde Coletiva. Cadernos Saúde Coletiva, 22(4), 419-427. doi: 10.1590/1414-462X201400040016

Milfont, T. L., \& Fischer, R. (2010). Testing measurement invariance across groups: Applications in cross-cultural research. International Journal of Psychological Research, 3(1), 111-121. doi: 10.21500/20112084.857

Moreira-Almeida A., Peres M. F., Aloe F., Lotufo Neto F., \& Koenig H. G. (2008). Versão em português da Escala de Religiosidade Duke DUREL. Revista de Psiquiatria Clínica, 35(1), 31-32. doi: 10.1590/S0101-60832008000100006

Nabipour, A. R., Khanjani, N., Nakhaeem N., Moradlou, H. Z., \& Sullman, M. J. M. (2015). The relationship between religion and the on-road behaviour of adolescents in Iran. Transportation Research Part F: Traffic Psychology and Behaviour, 29, 113-120. doi: 10.1016/j. trf.2015.01.009 
Panzini, R. G., Maganha, C., Rocha, N. S., Bandeira, D. R., \& Fleck, M. P. (2011). Validação brasileira do instrumento de qualidade de vida/ espiritualidade, religião e crenças pessoais. Revista de Saúde Pública, 45(1), 153-65. doi: 10.1590/S0034-89102011000100018

Saffari M., Zeidi I. M., Pakpour A. H., \& Koenig H. G. (2013). Psychometric properties of the Persian version of the Duke University Religion Index (DUREL): A study on Muslims. Journal of Religion and Health. 52(2), 631-41. doi: 10.1007/s10943-012-9639-0

Silva, D. G., Giordani, J. P., \& Dell'Aglio, D. D. (2017). Relações entre satisfação com a vida, com a família e com as amizades e religiosidade na adolescência. Estudos Interdisciplinares em Psicologia, 8(1), 38-54. doi: 10.5433/2236-6407.2016v8n1p38

Strelhow, M. R. W., Bedin, L. M., \& Sarriera, J. C. (2017). Children's Religious Coping Scale: Adaptation and psychometric properties. Paideia, 27(66), 107-116. doi:10.1590/1982-43272766201713

Strelhow, M. R. W., \& Henz, K. G. (2017). Spirituality and religiosity related to the well-being of children and adolescents: A theoretical and empirical approach. Em J. C. Sarriera \& L. M. Bedin (Eds). Psychosocial well-being of children and adolescents in Latin America: Evidence-based interventions (27-45). Editora Springer. doi: 10.1007/978-3-319-55601-7

Tabrizi, R., Akbari, M., Lankarani, K. B., Heydari, S. T., Masoudi, A., Shams, A. H., .. Peymani, P. (2017). Relationship between religion and school students' road behavior in southern Iran. Chinese Journal of Traumatology, 20(5), 264-269. doi: 10.1016/j.cjtee.2016.12.001

Taunay, T. C. E., Gondim, F. A. A., Macêdo, D. S., Moreira-Almeida, A., Gurgel, L. A., Andrade, L. M. S., \& Carvalho, A. F. (2012). Validação da versão brasileira da escala de religiosidade de Duke (DUREL). Archives of Clinical Psychiatry (São Paulo), 39(4), $130-135$. doi: 10.1590/S0101-60832012000400003

Thomas, K. H., McDaniel, J. T., Albright, D. L., Fletcher, K. L., \& Koenig, H. G. (2018). Spiritual fitness for military veterans: A curriculum review and impact evaluation using the Duke Religion Index (DUREL). Journal of Religion and Health, 57(3), 1168-1178. doi: 10.1007/ s10943-018-0597-z

Wang, Z., Rong, Y., \& Koenig, H. G. (2014). Psychometric properties of a Chinese version of the Duke University Religion Index in college students and community residents in China. Psychological Reports, 115(2), 427-443. doi: 10.2466/08.17.PR0.115c19z8

\section{Sobre os autores}

Miriam Raquel Wachholz Strelhow é psicóloga, pesquisadora de pós-doutorado na Universidade de São Paulo, USP, com bolsa concedida pela Fundação de Amparo à Pesquisa do Estado de São Paulo, FAPESP (processo 2018/02178-5). Doutorado e mestrado em Psicologia pela Universidade Federal do Rio Grande do Sul, UFRGS.

Jorge Castellá Sarriera é doutor em Psicologia, pesquisador 1A CNPq, professor no PPG em Psicologia e no PPG em Políticas Sociais e Serviço Sociais da UFRGS, coordenador do Grupo de Pesquisa em Psicologia Comunitária e prêmio pesquisador gaúcho em Ciências Humanas e Sociais em 2015. 